\title{
Biomaterial Surface Can Modify HUVEC Morphology and Inflammatory Response by Regulating MicroRNA Expression
}

\author{
Shuangying Gu, Baoxiang Tian, Weicong Chen, Yue Zhou \\ School of Biomedical Engineering and Med-X Research Institution, Shanghai Jiao Tong University, Shanghai, China \\ Email: yzhou2009@sjtu.edu.cn
}

How to cite this paper: Gu, S.Y., Tian, B.X., Chen, W.C. and Zhou, Y. (2017) Biomaterial Surface Can Modify HUVEC Morphology and Inflammatory Response by Regulating MicroRNA Expression. Journal of Biosciences and Medicines, 5, 8-16.

https://doi.org/10.4236/jbm.2017.512002

Received: August 1, 2017

Accepted: November 18, 2017

Published: November 21, 2017

\begin{abstract}
Vascular inflammation is an important process which contributes to the pathogenesis of many cardiovascular diseases, such as atherosclerosis. MicroRNAs (miRNAs) have been revealed as novel regulators of vascular inflammation. Prior researches had shown that alterations in gene expression of human umbilical vein endothelial cells (HUVECs) associated with topographic cues. Here, we showed that poly (dimethyl siloxane) (PDMS) substrate of $10 \mu \mathrm{m}$ width and $3 \mu \mathrm{m}$ depth parallel microgrooves on the surface could significantly upregulate the expression of anti-inflammatory microRNAs, miR-146a and miR-181b. In addition, the results also showed that TRAF6 and importin- $\alpha 3$, target of miR-146a and miR-181b, respectively, were both down-regulated $(P<0.05$ and $P<0.001$, respectively). The expression levels of the inflammation related proteins were all significantly decreased, including VCAM-1 $(P<0.05)$, ICAM-1 $(P<0.001)$, E-selectin $(P<0.001)$, and MCP-1 $(P<0.05)$. The adhesion of the mononuclear cell line, THP-1, was significantly decreased $(P<0.05)$. The results revealed that morphology modified HUVEC can modulate miR-146a and miR-181b and their downstream biological functions such as decreasing inflammation, suggesting that surface microtopology may affect vascular inflammation in the setting of cardiovascular disease. These interesting findings will facilitate the optimal design of microstructured materials in tissue engineering.
\end{abstract}

\section{Keywords}

HUVEC, Vascular Inflammation, MicroRNAs

\section{Introduction}

Vascular tissue engineering provides a promising approach in vascular disease. 
Previous research stresses the importance of biochemical modification of biomaterial in tissue repair and regeneration [1]. Recent studies have shown that physical factors played important role in regulating cellular behavior [2]. Mechanical forces associated with blood flow play an important role in regulating vascular signaling and gene expression in endothelial cells (ECs), for example, changes in shear stress result in differential expression of numerous microRNAs, triggering the balance between susceptibility and resistance [3] [4]. Recent study showed that the aligned nanofibers significantly induced neurite outgrowth and enhanced skin cell migration during wound healing compared to randomly oriented nanofibers, which shed light on the relative importance of the surface topography and chemical signaling in the guidance of different cell behavior [5]. In addition, biophysical cues, in the form of parallel microgrooves on the surface of cell-adhesive PDMS substrates, can replace the effects of small-molecule epigenetic modifiers and significantly improve reprogramming efficiency [6]. Alterations in gene expression of human vascular endothelial cells associated with nanotopographic cues [7].

Recent work has revealed that microRNAs (miRNAs) can also regulate vascular inflammation [8] [9]. Study from Fang et al. demonstrated that endothelial miR-10a expression is lower in the atherosusceptible regions of the aorta, suggesting the lower miR-10a expression may contribute to the pro-inflammatory endothelial phenotypes in atherosusceptible region [10]. A negative-feedback loop was identified in which miR-31 expression and miR-17-3p expression were induced by TNF- $\alpha$ in ECs to inhibit EC activation by reducing the expression of adhesion molecules [11]. In addition, miR-125 can reduce endothelial inflammation and atherosclerosis, and inhibit adherence of leukocytes to ECs [12]. Both miR-146a and miR-181b repress vascular inflammation through NF- $\mathrm{BB}$ signaling pathway, and overexpression of miR-146a and miR-181b down-regulated the level of inflammatory genes (VCAM-1, ICAM-1, E-Selectin, MCP-1) [13] [14].

Human cells in vivo are exposed to a topographically rich, 3-dimenisional environment, which provides extracellular cues initiating a cascade of biochemical signals resulting in changes in cell behavior. Topographic cues can significantly affect gene expression of human vascular endothelial cells, which can also affect the progress of cardiovascular diseases [7] [15]. To elucidate the role of biophysical factors in regulating endothelial functions, we used the cell-adhesive PDMS substrates with the surface of $10 \mu \mathrm{m}$ width and $3 \mu \mathrm{m}$ depth parallel microgrooves to physically change the shape of endothelial cell. We investigated whether the micro-patterned surface of the biomaterial substrate may induce specific microRNA expression to regulate cell inflammatory response.

\section{Methods}

\subsection{Cell Culture}

Human umbilical vein endothelial cells (HUVECs, Sciencell), passage 2 - 8, were 
cultured in ECM (Sciencell) with 5\% fetal bovine serum (FBS) and $1 \%$ penicillin/streptomycin (P/S), and $1 \%$ endothelial cell growth supplement (ECGS) at $37^{\circ} \mathrm{C}$ in a humidified $5 \% \mathrm{CO}_{2}$ atmosphere. Medium was changed every $2-3$ days. Subculture cells at $80 \%-90 \%$ confluence. The cells were seeded on the PDMS substrates for 3 days before proceeding to the following experiments.

\subsection{Fabrication and Characterization of PDMS Substrates}

To acquire patterned substrates with parallel microgrooves $(10 \mu \mathrm{m}$ in width and $3 \mu \mathrm{m}$ in depth), we used microfabrication technique to fabricate materials with desired surface topography. Poly (dimethyl siloxane) (PDMS) (Sylgard 184, Dow Corning, Midland, MI) was prepared according to the manufacturer's instruction. PDMS was spin-coated onto the patterned silicon wafers to achieve desired thickness $(\sim 250 \mu \mathrm{m})$, degassed under vacuum, and cured at $75^{\circ} \mathrm{C}$ for $1.5 \mathrm{~h}[6]$ [16]. The PDMS with micropatterned surface were removed from the template, cut into appropriate shapes and thoroughly cleaned by sonication, treated with Plasma Prep (11050Q-AX) to enhance the surface hydrophilicity, and coated with $2 \%$ gelatin for 1.5 hour to promote cell attachment. The images of the PDMS surface were collected by using scanning electron microscopy (SEM, JEOL JSM-5600).

\subsection{RNA Isolation and qRT-PCR Analysis}

The total RNA and microRNA were isolated using TRIzol ${ }^{\bullet}$ plus RNA Purification (No. 12183555, Invitrogen) and miRcute miRNA isolation kit (DP501, TIANGEN) respectively following the manufacture's instruction. For qRT-PCR, cDNA of mRNA synthesis was performed by using the FastQuant RT kit (with gDNase) (KR106, TIANGEN), the mRNA level was measurement using SuperReal PreMix Plus (SYBR Green) (FP209, TIANGEN). GAPDH was used as a normalization control. The sequences of the primers used for real-time PCR are listed in Table 1. cDNA of microRNA was synthesized by miRcute miRNA First-Strand cDNA Synthesis Kit (KR211, TIANGEN) and the quantitative real-time PCR was performed by miRcute miRNA qPCR Detection Kit (SYBR Green) (FP411, TIANGEN) according to the manufacturer's instruction. RNU6-2 was used as a normalization control in all miRNA measurements. Primers used were listed as shown in Table 2. The qRT-PCR was run on Applied Biosystems 7900HT Fast Real-Time PCR system (ABI). And the qRT-PCR data were analyzed using the comparative $2^{-\triangle \Delta C T}$ method.

Table 1. The primers of mRNAs used for $\mathrm{qRT}-\mathrm{PCR}$.

\begin{tabular}{cll}
\hline Primers & \multicolumn{1}{c}{ Forward Primer } & \multicolumn{1}{c}{ Reverse Primer } \\
\hline GAPDH & 5' GGG AAG GTG AAG GTC GGA GT & 5' GGG GTC ATT GAT GGC AAC A \\
VCAM-1 & 5' AAA GGG AGC ACT GGG TTG & 5' GCA CAG GAG TCT GAT GAA CA \\
ICAM-1 & 5' GGC ATT GTC CTC AGT CAG AT & 5' TCC TTC CTC TTG GCT TAG TC \\
E-Selectin & 5' CAA CAC CCA TCA CCA CTT C & 5' CTT TCC CTT CAT TAG CCA AC \\
MCP-1 & 5' CCG AAG ACT TGA ACA CTC AC & 5' CTG GGG AAA GCT AGG GGA A \\
\hline
\end{tabular}


Table 2. The primers of microRNAs used for qRT-PCR.

\begin{tabular}{cc}
\hline Primers & Forward Primer \\
\hline hsa-miR-146a-5p & 5' UGA GAA CUG AAU UCC AUG GGU U \\
hsa-miR-181b-5p & 5' AAC AUU CAA CGC UGU CGG UGA GU \\
\hline
\end{tabular}

\subsection{Immunostaining}

For immunostaining, the actin-cytoskeleton and cell membrane was stained by antibody of FITC-phalloidin (FITC, AAT Bioquest) and $\beta$-catenin (Abcam) respectively, Nuclei were stained with 4',6-diamino-2-phenylindole (DAPI, Beyotime Biotechnology). The microscopic images acquired by Confocal Laser Scanning Microscope TCS SP5II (Leica).

\subsection{Monocyte Adhesion Assay}

$50 \mathrm{ng} / \mathrm{ml}$ LPS was added to the ready HUVECs for 5 hours. THP-1 cells (ATCC) were washed with serum-free RPMI 1640 medium and suspended in medium with $4 \mu \mathrm{M}$ of Calcein AM (1631214; Invitrogen). Cells were kept in an incubator for 30 minutes. The labeling reaction was stopped by adding the cell growth medium, and cells were washed with growth medium twice and resuspended in growth medium. After 5 hours of LPS treatment, HUVECs were washed once with THP-1 cell growth medium, and Calcein AM-loaded THP-1 cells were added to each well. After 1 hour of incubation, non-adherent cells were removed carefully. Adherent cells were gently washed with pre-warmed RPMI 1640 medium 3 times. The number of THP-1 cells per view was quantified from randomly acquired images.

\subsection{Statistical Analysis}

Unless otherwise indicated, data represent the mean of at least 3 independent experiments and error bars represent the standard error of the mean (SEM). For all cases, p-values less than 0.05 were considered statistically significant. In all figures, ${ }^{*}$ represent $P<0.05$, ${ }^{* *}$ represent $P<0.01$, and ${ }^{* * *}$ represent $P<0.001$. GraphPad Prism 6.0 software was used for all statistical evaluations.

\section{Results}

\subsection{Characterization of PDMS Substrates and HUVECs Morphology}

In order to increase the cell attachment and biocompatibility of the biomaterial, $2 \%$ gelatin was used to coat the PDMS substrates surface to improve the hydrophilicity. After coating with gelatin, the hydrophilicity of PDMS surfaces was determined by water contact angle measurements. The results are shown in (Figure 1) and Table 3, which indicated that gelatin coating for 1 hour can significantly increase the hydrophilicity of the PDMS substrates, and no significant difference was observed between the flat surface and microgrooved surface. 


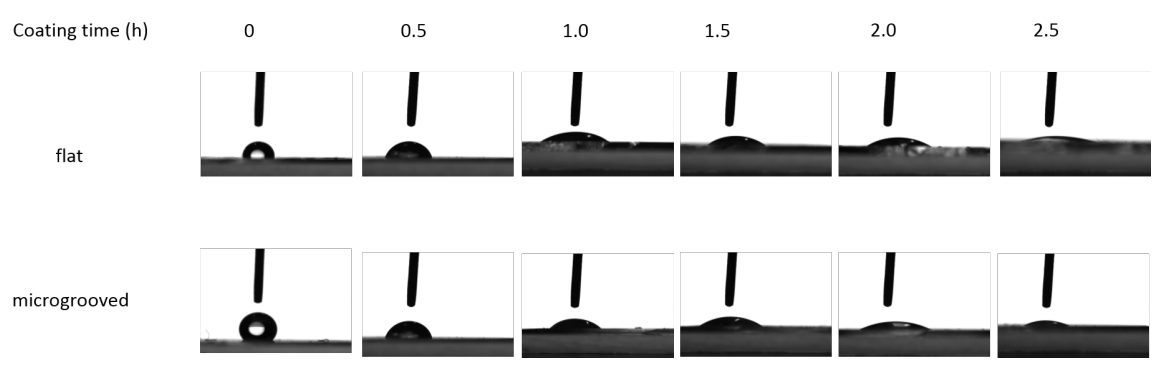

Figure 1. The water contact angle of PDMS substrates coated with gelatin for different time. The water contact angle of both flat and microgrooved surface of PDMS substrates before and after coating with gelatin. Coating time was optimized from 0.5 to $2.5 \mathrm{~h}$.

Table 3. Contact angle of both flat and grooved surfaces of PDMS substrates.

\begin{tabular}{ccccccc}
\hline Coating time (h) & & & & & & \\
Pattern & 0 & 0.5 & 1 & 1.5 & 2 & 2.5 \\
\hline flat & 134.7 & 83.2 & 43.6 & 37.0 & 34.0 & 12.1 \\
microgrooved & 153.5 & 88.8 & 36.4 & 36.3 & 33.8 & 25.7 \\
\hline
\end{tabular}

To observe the HUVEC morphology modified by micro-patterned substrates, HUVECs were seeded onto flat poly (dimethyl siloxane) (PDMS) substrates and the micro-patterned one fabricated with a $10 \mu \mathrm{m}$ parallel grooves in width and 3 $\mu \mathrm{m}$ in depth. SEM characterized the three-dimensional structure of PDMS substrates (Figure 2(a)), HUVECs morphology was demonstrated by FITC-phalloidin, DAPI, and $\beta$-catenin staining (Figure 2(b)). As expected, phalloidin and $\beta$-catenin staining indicated that microgroove substrates had a pronounced effect on HUVEC morphology. In general, cells grown in microgroove PDMS substrates exhibited a more elongated morphology followed the guidance of parallel grooves, and the stress fibers were also parallel to the axis of groove alignment. The nuclear elongation was also observed in some cells.

The effect of the microgrooved surface on modifying the morphology of HUVEC shown here was selected from a series designed width based on our preliminary experiments. The width should be fine-tuned according to the size of the cells culture on it. In case of HUVEC, microgrooves with $10 \mu \mathrm{m}$ in width were suitable to elongate the cells regardless of seeding density.

\subsection{The Expression of miR-146a and miR-181b Was Induced in HUVECs Cultured on Microgrooved Surface}

Previous studies have shown that topographic cues can significantly affect cell gene and microRNA expression levels in human vascular endothelial cells. To test whether our designed microgrooved PDMS substrates can also affect anti-inflammatory microRNAs, HUVECs were cultured on grooved and flat PDMS substrates biomaterial, and then the microRNAs were collected to examine the expression of miR-146a and miR-181b. The results showed that microgrooved PDMS substrates significantly increased the expression of miR-146a $(P<0.05)$ and miR-181b $(P<0.001)$ (Figure 3(a)). TRAF6 and importin- $\alpha 3$ were known 


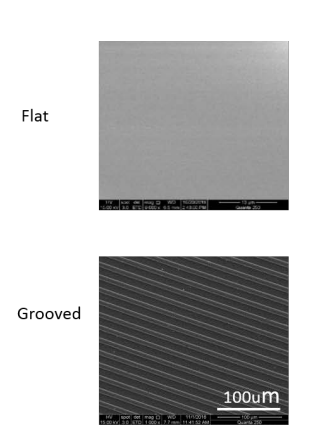

(a)

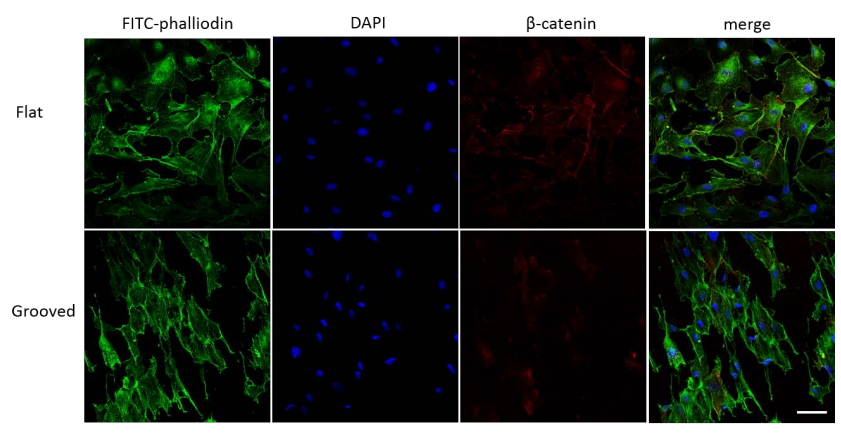

(b)

Figure 2. HUVEC morphology on different PDMS substrates. (a) Scanning electron micrograph (SEM) images of PDMS substrateswith flat or microgrooved surface. The image of the microgrooved PDMS substrates with a $10 \mu \mathrm{m}$ parallel grooves width and 3 $\mu \mathrm{m}$ in depth; (b) The confocal image of HUVECs cultured on flat and grooved PDMS substrates. Cells were stained with phalloidin (green), DAPI (blue), and anti- $\beta$-catenin antibody (red). Scale bar, $75 \mu \mathrm{m}$.

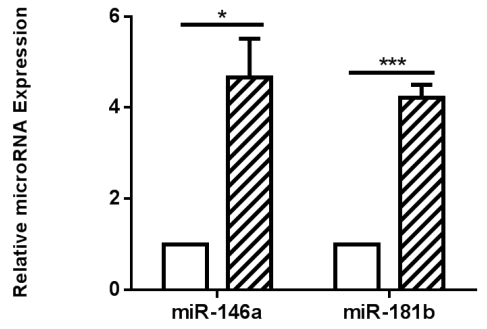

(a)
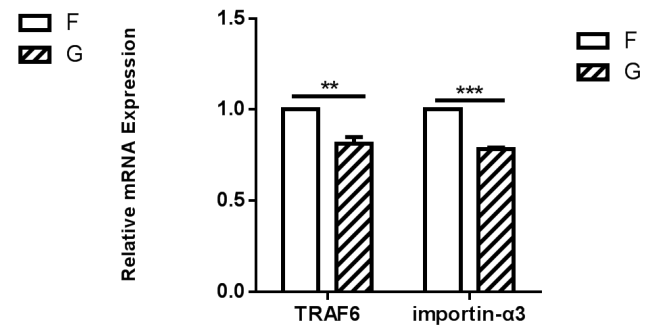

(b)

Figure 3. The expression of the microRNAs and their targets genes in the HUVECs on different PDMS substrates. (a) qRT-PCR results of the expression of the anti-inflammatory miRNAs (miR-146a, miR-181b) in HUVEC (normalized to U6 expression). Data shown was a representative of 3 parallel experiments. $\left({ }^{\star} P<0.05,{ }^{* \star} P<0.01,{ }^{* * *} P<0.001\right)$; (b) qRT-PCR results of the expression of TRAF6 and importion- $\alpha 3$ in HUVEC (normalized to GAPDH expression). Data shown was a representative of 3 parallel experiments. $\left({ }^{\star} P<0.05,{ }^{* *} P<0.01,{ }^{* * *} P<0.001\right)$.

as the target genes of miR-146a and miR-181b, respectively. We further tested the mRNA levels of TRAF6 and importin- $\alpha 3$ and found both were significantly suppressed on grooved PDMS substrates $(P<0.01$ and $P<0.001$, respectively, Figure 3(b)). These results consistently supported our hypothesis that biomaterial substrate may induce specific microRNA expression including those regulating cell inflammatory response.

\subsection{Up-Regulation of miR-146a and miR-181b Modulates Inflammatory Response in HUVECs}

In response to endothelial activation, adhesion molecules, such as VCAM-1, ICAM-1, and E-selectin, act to initiate, promote, and sustain leukocyte attachment to the vascular endothelium. To assess whether microgrooved surface might suppress the expression of these specific proteins to inhibit the inflammation, the expression levels of VCAM-1, ICAM1, SELE (E-Selectin), and MCP-1, 
were measured in LPS-stimulated HUVECs by qRT-PCR. The results showed that the level of those inflammatory genes was all significantly decreased on microgrooved PDMS substrates relative to the flat substrates (VCAM-1, $P<0.05$; ICAM1, $P<0.001$; SELE (E-Selectin), $P<0.001$, and MCP-1, $P<0.05$, Figure 4(a)), suggesting that the endothelial inflammatory response was suppressed.

To further confirm the functional consequence of the decreased expression of the inflammatory genes, we employed an in vitro cell adhesion assay to assess leukocyte-endothelium interactions. Mononuclear cell line THP-1 was used in this assay. The adhesion of THP-1 cells to HUVECs on microgrooved PDMS substrates was markedly decreased ( $P<0.05$, Figure 4(b) and Figure 4(c)).

Taken together, these findings suggest that microgrooved PDMS substrates can inhibit the HUVECs inflammatory response by induction of the expression of anti-inflammatory microRNAs.

\section{Conclusions}

We demonstrated that microgrooved PDMS substrates can change the morphology of HUVEC by specifically designing the width of the grooves to $10 \mathrm{um}$. When the morphology of HUVEC was changed, the expression of miR-146a and miR-181b could be significantly induced at the same time, and their target genes

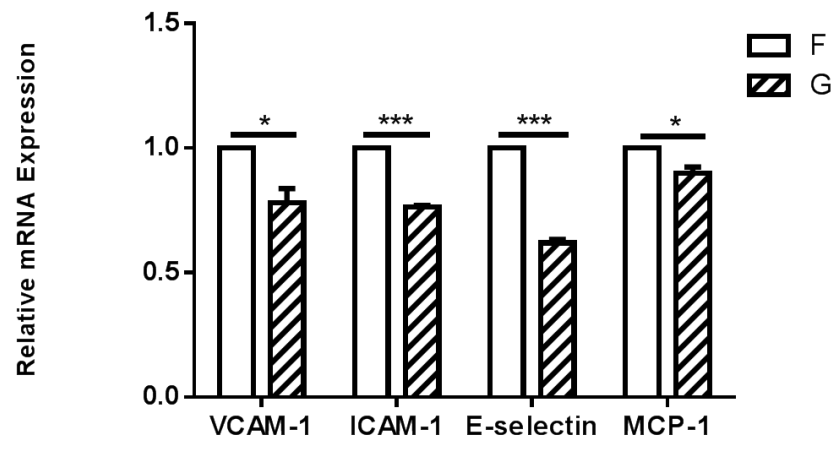

(a)

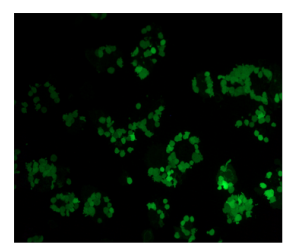

flat

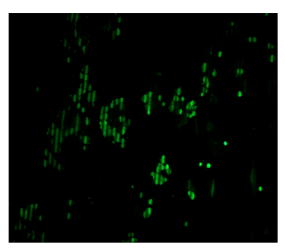

grooved

(b)

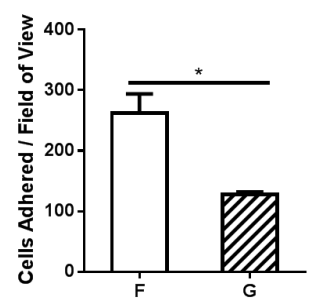

(c)

Figure 4. Comparison of the inflammatory response of the HUVECs on different PDMS membranes (a) qRT-PCR results of the expression levels of the inflammatory genes (VCAM-1, ICAM-1, E-selectin, MCP-1) in HUVECs (normalized to GAPDH). Data shown was a representative of 3 parallel experiments. $\left({ }^{\star} P<0.05,{ }^{* *} P<0.01,{ }^{* * *} P<0.001\right)$; (b) The confocal image of the adhesion of THP-1 to LPS-treated HUVECs grown on PDMS substrates with flat or microgrooved surface; (c) The quantitation of the THP-1 adhered to LPS-treated HUVECs grown on PDMS substrates with flat or microgrooved surface. 
down-regulated accordingly. Furthermore, the inflammatory response of HUVECs could be inhibited. As a favorable consequence, the specially designed microgrooves may be considered to be used in manufacturing tissue engineered blood vessels.

\section{Acknowledgements}

This work was supported by the Science and Technology Commission of Shanghai Municipality (16140901900).

\section{References}

[1] Dai, M., Zheng, X., Xu, X., Kong, X., Li, X., Guo, G., et al. (2010) Chitosan-Alginate Sponge: Preparation and Application in Curcumin Delivery for Dermal Wound Healing in Rat. Journal of Biomedicine \& Biotechnology, 2009, 595126.

[2] Chien, S. (2007) Mechanotransduction and Endothelial Cell Homeostasis: The Wisdom of the Cell. AJP Heart \& Circulatory Physiology, 292, 135-180.

[3] Ando, J. and Yamamoto, K. (2011) Effects of Shear Stress and Stretch on Endothelial Function. Antioxidants \& Redox Signaling, 15, 1389-1403. https://doi.org/10.1089/ars.2010.3361

[4] Neth, P., Nazari-Jahantigh, M.,Schober, A. and Weber, C. (2013) MicroRNAs in Flow-Dependent Vascular Remodelling. Cardiovascular Research, 99, 294-303. https://doi.org/10.1093/cvr/cvt096

[5] Patel, S., Kurpinski, K., Quigley, R., Gao, H.F., Hsiao, B.S., Poo, M.M., et al. (2007) Bioactive Nanofibers: Synergistic Effects of Nanotopography and Chemical Signaling on Cell Guidance. Nano Letters, 7, 2122-2128. https://doi.org/10.1021/nl071182z

[6] Downing, T.L., Soto, J., Morez, C., Houssin, T., Fritz, A. Yuan, F., et al. (2013) Biophysical Regulation of Epigenetic State and Cell Reprogramming. Nature Materials, 12, 1154-1162. https://doi.org/10.1038/nmat3777

[7] Gasiorowski, J.Z., Liliensiek, S.J., Russell, P., Stephan, D.A., Nealey, P.F. and Murphy, C.J. (2010) Alterations in Gene Expression of Human Vascular Endothelial Cells Associated with Nanotopographic Cues. Biomaterials, 31, 8882-8888. https://doi.org/10.1016/j.biomaterials.2010.08.026

[8] Yamakuchi, M. (2012) MicroRNAs in Vascular Biology. International Journal of Vascular Medicine, 2012, 794898. https://doi.org/10.1155/2012/794898

[9] Ono, K., Kuwabara, Y. and Han, J. (2011) MicroRNAs and Cardiovascular Diseases. MicroRNAs in Medicine. FEBS Journal, 278, 1619-1633. https://doi.org/10.1111/j.1742-4658.2011.08090.x

[10] Fang, Y. and Catravas, J. (2010) MicroRNA-10a Regulation of Proinflammatory Phenotype in Athero-Susceptible Endothelium in Vivo and in Vitro. Proceedings of the National Academy of Sciences of the United States of America, 107, 13450-13455. https://doi.org/10.1073/pnas.1002120107

[11] Suárez, Y., Wang, C., Manes, T.D. and Pober, J.S. (2010) Cutting Edge: TNF-Induced MicroRNAs Regulate TNF-Induced Expression of E-Selectin and Intercellular Adhesion Molecule-1 on Human Endothelial Cells: Feedback Control of Inflammation. Journal of Immunology, 184, 21-25.

[12] Dong, L.I., Yang, P., Rui, Y. and Yuan, W. (2010) MicroRNAs Inhibit Endothelin-1 Expression in Vascular Endothelial Cells. Journal of Hypertension, 128, 1646-1654. 
[13] Sun, X., Icli, B., Wara, A.K., Belkin, N., He, S., Kobzik, L., et al. (2012) MicroRNA-181b Regulates nf-kb-Mediated Vascular Inflammation. Journal of Clinical Investigation, 122, 1973-1990.

[14] Cheng, H.S., Sivachandran, N., Lau, A., Boudreau, E., Zhao, J.L., Baltimore, D., et al. (2013) MicroRNA-146 Represses Endothelial Activation by Inhibiting Pro-Inflammatory Pathways. EMBO Molecular Medicine, 5, 1017-1034. https://doi.org/10.1002/emmm.201202318

[15] Biela, S.A., Su, Y., Spatz, J.P. and Kemkemer, R. (2009) Different Sensitivity of Human Endothelial Cells, Smooth Muscle Cells and Fibroblasts to Topography in the Nano-Micro Range. Acta Biomaterialia, 5, 2460-2466.

https://doi.org/10.1016/j.actbio.2009.04.003

[16] Downing, T.L., Soto, J., Morez, C., Houssin, T., Fritz, A., Yuan, F., et al. (2013) Biophysical Regulation of Epigenetic State and Cell Reprogramming. Nature Materials, 12, 1154-1162. https://doi.org/10.1038/nmat3777 